\title{
Le modèle espagnol de direction
}

\section{Miguel Recio Muñiz}

Traducteur : Claudine Adam

\section{(2) OpenEdition \\ Journals}

Édition électronique

URL : http://journals.openedition.org/ries/4191

DOI : 10.4000/ries.4191

ISSN : 2261-4265

\section{Éditeur}

Centre international d'études pédagogiques

\section{Édition imprimée}

Date de publication : 1 décembre 1994

Pagination : 59-65

ISSN : 1254-4590

\section{Référence électronique}

Miguel Recio Muñiz, "Le modèle espagnol de direction », Revue internationale d'éducation de Sèvres [En ligne], 04 | 1994, mis en ligne le 17 avril 2015, consulté le 07 janvier 2020. URL : http://

journals.openedition.org/ries/4191; DOI : 10.4000/ries.4191

Ce document a été généré automatiquement le 7 janvier 2020

(c) Tous droits réservés 


\title{
Le modèle espagnol de direction
}

\author{
Miguel Recio Muñiz \\ Traduction : Claudine Adam
}

1 Le système éducatif espagnol est plongé dans de profondes transformations qui concernent deux domaines principaux :

- la construction de la nouvelle structure du système éducatif, d'une part - nouvelles étapes éducatives, prolongation jusqu'à seize ans de l'enseignement obligatoire, définition de l'étape éducative de l'enseignement secondaire obligatoire (de 12 à 16 ans) comme un enseignement compréhensif, c'est-à-dire commun et ouvert à la diversité, création d'une nouvelle formation professionnelle à base de modules, proche des besoins professionnels modernes et des entreprises ;

- l'implantation de nouveaux principes en matière de pédagogie, d'organisation et de fonctionnement, d'autre part. Ces principes résument les fondements essentiels du modèle éducatif espagnol et, en même temps, organisent son application concrète dans la vie quotidienne des établissements scolaires.

2 Tels sont les deux objectifs de la LOGSE (loi d'aménagement général du système éducatif) dont la mise en place se fera progressivement de 1990 à l'an 2000. Comment vit-on actuellement en Espagne l'implantation de la réforme ? En quoi cette importante réforme éducative affecte-t-elle la direction des établissements? Et quel rôle peut jouer la direction des établissements dans la réalisation des objectifs de la réforme? Il est clair que des modifications aussi profondes entraînent nécessairement des changements substantiels pour les équipes de direction.

\section{Les relations avec les différents partenaires de la communauté éducative}

3 Lorsqu'en 1979 et 1980, juste après l'approbation de la Constitution espagnole, les autorités éducatives et politiques commencent à rédiger les lois qui vont normaliser son application dans le domaine de l'éducation, l'un des principaux sujets d'intérêt, et bientôt de polémique, est la réglementation des relations entre le chef d'établissement 
et l'équipe de direction dans son ensemble, d'une part, et la communauté éducative et l'administration, d'autre part. La démocratie devait-elle entrer dans les écoles et les lycées avec l'élection du directeur ainsi que de l'équipe de direction et d'administration par les parents, les élèves, les professeurs et les représentants des personnels de l'administration et des services éducatifs? L'élection du directeur et de l'équipe de direction était-elle la manifestation la plus importante de participation et contrôle dans la gestion des établissements, tels qu'ils étaient inscrits dans la Constitution?

4 La polémique fut telle que deux groupes se formèrent : d'un côté, ceux qui défendaient une plus grande autonomie des établissements et un rôle pédagogique plus important du Claustro $^{1}$ des professeurs et choisissaient d'élire le directeur et l'équipe de direction ; de l'autre, ceux qui défendaient une diminution de l'autonomie des établissements et un poids accru des contenus administratifs et bureaucratiques dans le travail de direction des établissements et choisissaient la désignation du directeur à partir, principalement, de l'opinion de l'administration.

Ces deux modèles ont pris forme dans des partis politiques aux programmes éducatifs difficilement conciliables, ce qui ne les a pas empêchés d'appliquer et de développer, chacun à son tour, leurs points de vue au cours des législatures où ils gouvernaient.

6 Bien qu'elles aient concerné des droits fondamentaux, les lois éducatives ont été parmi les seules (et même, au début, l'unique exemple) à être élaborées sans consensus entre les deux grandes formations politiques de droite et de gauche. Pour les conservateurs, le chef d'un établissement public devait continuer à être, avant tout, un représentant de l'administration éducative ; c'est donc à elle que devait fondamentalement revenir le choix de ce responsable. Mais le même camp changeait d'arguments lorsqu'il parlait des directeurs des nombreux établissements privés - subventionnés avec les deniers publics -, que l'on trouve en Espagne. En tous cas, les partis de gauche considéraient que cette dépendance du chef d'établissement à l'administration éducative était un signe du maintien de la présence de l'État dans une sphère qui devait être du domaine public: l'école. Rendre publics les établissements exigeait de rompre avec la dépendance par rapport à l'état et la meilleure façon de le faire était de couper le lien chef d'établissement - administration éducative.

7 C'est ainsi qu'à partir de 1979, on commença à promulguer des lois qui augmentaient peu à peu le poids des représentants des différents secteurs de la communauté éducative (parents, élèves, professeurs, personnels de l'administration et des services éducatifs, mairie) dans le choix du directeur et de l'équipe de direction; et, en 1985, la loi du droit à l'éducation (LODE) réserva l'élection du directeur et de toute l'équipe de direction au seul conseil scolaire de l'établissement, cet organe où parents, élèves, personnels de l'administration, des services éducatifs et porte-parole de la mairie élisent leurs représentants (leur nombre étant égal à celui des représentants élus par les professeurs). Ce modèle est en vigueur depuis 1989 et, au bout de huit ans, on peut tirer quelques conclusions sur sa pertinence. En premier lieu, il est indéniable que l'intérêt et la participation de la communauté éducative à la vie de l'établissement, à son contrôle et à sa gestion ont augmenté dans les établissements d'enseignement publics et privés; mais, en même temps que s'est accrue la participation, est né un sentiment de désenchantement et de frustration causé, principalement, par un manque d'autonomie réelle et de ressources permettant de mener à bien les projets qui étaient approuvés. Dans la plupart des cas, la participation des différents secteurs n'a pas abouti à un projet commun car on n'avait pas les moyens de le développer. Dans 
certains cas, cette situation a donné lieu à des prises de position antagoniques entre les différents secteurs de la communauté éducative, que professeurs et directeurs ont ressenties parfois comme une immixtion dans leur travail. À ces problèmes s'ajoute le reflux de la participation dans les écoles allant de pair avec la crise de la participation en général, ce qui, bien souvent, a entraîné une diminution de la candidature des professeurs au poste de directeur; au cours de cette année scolaire, la moitié des directeurs à peine a été élue par les conseils scolaires, les autres ont été désignés par l'administration elle-même.

Parallèlement à ces faits et à ces tendances, suffisants en eux-mêmes pour entraîner une révision du système actuel de nomination des directeurs, il faut remarquer qu'à partir de 1990, le gouvernement a adopté une série de mesures visant à doter les établissements éducatifs d'une plus grande autonomie réelle. C'est ainsi qu'en 1990 est approuvée la norme d'autonomie économique des établissements, et en octobre de cette même année est votée la LOGSE déjà citée, la loi de réforme éducative qui commence à être implantée dans les établissements du second cycle à partir de l'année 1992-1993. Cette loi, qui attribue une grande autonomie pédagogique aux établissements, entraîne un effet de choc pour ce qui concerne la nomination et les tâches des équipes de direction.

\section{Direction des établissements et réforme du système éducatif}

9 Sans chercher à transformer les systèmes de participation et de gestion des établissements scolaires, la nouvelle loi de réforme éducative modifie de façon substantielle la précédente situation des équipes de direction, leur rôle et leurs fonctions. Assumer la direction d'un établissement, ce n'est plus affronter la simple gestion d'un modèle éducatif connu, gestion où les principaux problèmes, si compliqués soient-ils, sont d'ordre administratif. Avec la nouvelle loi, ce sont les tâches purement pédagogiques, académiques et enseignantes qui vont acquérir un rôle prépondérant dans le travail de l'équipe de direction, au détriment des tâches d'administration et de gestion. À la norme sur l'autonomie pédagogique et curriculaire (1990 et 1992) s'ajoute celle qui existe déjà sur l'autonomie économique (1990) et, plus récemment (1994), les normes concernant l'autonomie dans le domaine de l'organisation et du fonctionnement. Des établissements dotés d'autonomie pour l'économie, la pédagogie, l'organisation et le fonctionnement, tel est le nouveau cadre dans lequel il convient de redéfinir les fonctions et les tâches des équipes de direction. C'est en ces termes qu'est posé le débat: l'élection de l'équipe de direction est-elle le mécanisme adéquat pour permettre aux établissements de se doter de personnes capables d'affronter les défis qui naîtront de l'ampleur de leur autonomie ? Ou, peut-être, en simplifiant le débat: participation ou efficacité ? Élection ou sélection? Représentant de l'établissement face à l'administration éducative ou représentant de l'administration dans l'établissement ?

Le ministère de l'éducation espagnol, impliqué dans ce débat, a commencé à faire quelques pas (création du poste d'administrateur pour décharger les directeurs des tâches de gestion administrative, incitation à l'utilisation d'outils informatiques avec applications administratives et comptables) et à présenter des réflexions et des propositions. La première de toutes consiste à ouvrir le débat sur la nécessité de revoir et d'ajuster les caractéristiques d'une équipe de direction élue, qui doit aussi faire face 
à une autonomie qui l'amènera à prendre des décisions de grande importance pour l'établissement en harmonie et en corrélation avec les instances supérieures de l'administration éducative. Quelles doivent être les caractéristiques d'une équipe de direction en charge d'un établissement scolaire où l'on est en train d'implanter la réforme éducative? Quelles sont ses tâches et ses fonctions principales?

Les équipes de direction des écoles et des lycées sont composées au minimum d'un chef d'établissement, d'un responsable des études (ayant des compétences importantes dans le domaine des décisions pédagogiques), et d'un secrétaire (ayant des compétences importantes dans le domaine des décisions économiques). Les établissements de l'enseignement secondaire ont aussi un directeur adjoint (ou responsable des activités extrascolaires) et un responsable des études adjoint, chargés des différents niveaux ou cycles de l'établissement: enseignement secondaire obligatoire, baccalauréat ou formation professionnelle. Donc, le chef d'établissement se présente, seul, devant le conseil des professeurs (qu'il informe de ses intentions) et devant le conseil scolaire, afin d'obtenir leurs votes pour un programme de direction de l'établissement sur trois ans présentant les réflexions du candidat sur les problèmes de l'établissement et leurs solutions. S'il est élu - il ne peut l'être qu'à la majorité absolue -, il présente à l'élection son équipe de direction. S'il n'y a aucun candidat dans l'établissement (c'est ce qui arrive ces derniers temps dans $50 \%$ des cas) ou si le candidat n'obtient pas la majorité absolue, l'administration éducative départementale désigne un directeur provisoire pour un an.

12 Le chef d'établissement type est généralement un homme, bien que cette profession soit essentiellement féminine ; c'est davantage le cas des directeurs élus pour trois ans que de ceux désignés par l'administration. Il a une certaine ancienneté, une expérience enseignante et il réussit, relativement facilement, à trouver des candidats pour les autres postes de l'équipe de direction. Ses compétences le transforment en chef $d u$ personnel de l'établissement (il a en premier lieu la responsabilité du contrôle des absences, de l'attribution des horaires, etc.), en président de tous les organes de gouvernement collégiaux de l'établissement et en responsable ultime de toutes les décisions à caractère administratif, économique, pédagogique et d'organisation adoptées dans l'établissement. De par ses fonctions (il doit respecter et faire respecter les lois), il est autant un représentant de l'administration dans l'établissement qu'un représentant de l'établissement face à l'administration (il doit exécuter les décisions adoptées par les organes collégiaux de l'établissement) - situation de médiation exigeant $\mathrm{du}$ doigté et une vision d'ensemble des problèmes. Dans le domaine économique, le chef d'établissement doit défendre un budget devant le conseil scolaire ; si ce budget est approuvé, le directeur aura une complète autonomie pour les dépenses et les recettes ; il ne devra rendre de comptes qu'au conseil scolaire. Ce budget (qui est essentiellement établi à partir de la subvention de l'administration) fixe les ressources attribuées aux départements, les aides pour telle ou telle activité extrascolaire, les priorités concernant les travaux ou les achats, etc.

13 En ce qui concerne l'organisation et le fonctionnement de l'établissement, la direction de l'établissement propose - et le conseil scolaire approuve - l'horaire général (heures d'ouverture de l'établissement), décide l'ouverture ou non de l'établissement l'aprèsmidi pour réaliser des activités extrascolaires et répondre aux demandes de la communauté résidant près de l'établissement, enfin élabore les horaires individuels des 
professeurs et des élèves, s'occupe de l'admission des nouveaux élèves : en bref, elle a toute liberté pour les critères d'organisation des activités de l'établissement.

Mais c'est dans le domaine pédagogique que son rôle est le plus important, en raison de l'autonomie plus grande de l'établissement dans ce secteur. L'équipe de direction a la responsabilité et l'initiative de l'élaboration des deux documents où sont recueillis les objectifs éducatifs de l'établissement : d'une part, le projet éducatif de l'établissement (ensemble d'idées et de décisions, assumées par toute la communauté éducative scolaire, se rapportant aux options éducatives de base et à l'organisation générale de l'établissement), et, d'autre part, les projets curriculaires des cycles existant dans l'établissement (enseignements mis en œuvre dans l'établissement, en vertu de l'ouverture et de la flexibilité du curriculum). Ces documents développent les contenus et les cours dispensés, les critères d'évaluation, le passage et les diplômes des élèves, la méthodologie suivie, la répartition des ressources humaines et matérielles en vue d'apporter un soutien aux élèves ayant des difficultés d'apprentissage, les matières optionnelles proposées par l'établissement, la réalisation des tâches d'orientation et de tutorat, les critères permettant d'évaluer les objectifs fixés par l'établissement, etc. La tâche de l'équipe de direction est de coordonner les projets et d'encourager leur développement.

La réalisation de toutes ces tâches, en raison de la marge d'autonomie qu'elles impliquent est en train de changer le sens de la participation de la communauté éducative, le rôle de l'administration et, ce qui est plus important, le travail même des professeurs. Les établissements commencent à se différencier par le biais des options définies par les décisions du conseil des professeurs et du conseil scolaire.

\section{Difficultés et conséquences}

Ce rôle nouveau du chef d'établissement et de l'équipe de direction se heurte à diverses difficultés.

D'abord, le problème posé par les nouvelles fonctions assignées au directeur: cela signifie que certains directeurs, tout en étant de bons gestionnaires ou administrateurs, manquent de compétence pour affronter les tâches pédagogiques.

Deuxièmement, les difficultés qui relèvent des résistances de la communauté éducative elle-même. Tout ce qui est "ouvert», impliquant une prise de décisions, peut faire naître l'insécurité qui peut elle-même se transformer en méfiance vis-à-vis de ceux qui doivent prendre ces décisions. C'est pourquoi les parents peuvent préférer un curriculum fermé à un curriculum ouvert qui pourrait être mal complété par un établissement. Les professeurs eux-mêmes ressentent souvent comme une charge cette nouvelle obligation de prendre des décisions au sujet du curriculum et, surtout, voient comme une intromission dans leur liberté d'enseignant toutes les indications à caractère général contenues dans la partie commune du projet curriculaire. Il s'agit là d'un élément nouveau, à valeur normative, qui s'interpose entre leur liberté de professeurs et les indications énoncées dans les lois éducatives. Les principales issues à ces problèmes sont le dialogue avec tous les membres de la communauté éducative et la formation spécifique.

Troisièmement, les problèmes posés par ceux qui, tout en assumant les projets pédagogiques, se préoccupent avant tout de l'élaboration de ces documents (en 
particulier le projet curriculaire) et les transforment rapidement en documents bureaucratiques qui doivent être prêts à une date déterminée. Face à ce phénomène, il faut, même au prix d'un contrôle administratif plus souple, accorder plus d'importance au processus d'élaboration des documents en lui-même et aux valeurs que les professeurs lui attribuent : réflexions sur les raisons et les conséquences de leur travail, travail en équipe, dialogue avec les collègues sur les aspects pédagogiques et échange de documents et d'expériences, etc. Quatrièmement, les problèmes causés par l'absence d'aide pour réaliser ces tâches; aide jusqu'alors apportée par ceux qui étaient les principaux alliés des professeurs en général et des responsables en particulier. Il n'existe pas en effet de culture sociale sur laquelle puissent s'appuyer l'école et ses responsables face aux nouvelles responsabilités qu'ils doivent affronter. Les éditeurs n'offrent pratiquement pas de manuels scolaires pour l'enseignement secondaire et il est difficile de trouver à l'Université des spécialistes capables de donner des orientations, au-delà de la simple définition des modèles théoriques, utiles aux professeurs. Les familles ont du mal à appréhender la structure du nouveau système ; on leur demande maintenant beaucoup plus qu'un choix entre lettres et sciences (dès 12 ans il y a des options, et à 15 ans il faut choisir sept des dix matières).

Cependant, les conséquences, bien que lentes, sont très positives. Les équipes de direction se concentrent sur leurs réels domaines de compétence, en débattant avec leurs collègues sur le contenu et pas seulement sur les aspects administratifs. Les établissements gagnent en unité et en efficacité. Les ressources sont mieux administrées car elles visent un seul but et, en général, les taux d'échec scolaire diminuent. Voici ce qui distingue un bon établissement d'un mauvais : les professeurs et les élèves y sont, individuellement, très semblables, mais le rôle du chef d'établissement et de l'équipe dirigeante y sont totalement différents - dans un bon établissement, la dimension pédagogique est l'essentiel du travail et on y subordonne les décisions d'organisation et de fonctionnement; dans un mauvais établissement, cette dimension n'est pas abordée ou secondaire face aux aspects purement administratifs.

\section{NOTES}

1. Claustro : conseil des professeurs regroupant tous les enseignants d'un établissement (NdIR).

\section{RÉSUMÉS}

Comment la réforme globale du système éducatif espagnol, votée en 1990, affecte-t-elle le mode de direction des établissements? Les spécificités de ce fonctionnement (équipe de direction élue, 
implication de la communauté éducative) sont analysées dans le cadre de la nouvelle autonomie, pédagogique et financière, accordée aux établissements.

INDEX

Index géographique : Espagne

Mots-clés : autonomie, chef d'établissement, établissement d'enseignement, personnel d'encadrement, réforme

\section{AUTEURS}

\section{MIGUEL RECIO MUÑIZ}

Conseiller technique, ministerio de educación y ciencia, Madrid, Espagne 